\title{
Effect of Particle Morphology on the Ripening of Supported Pt Nanoparticles
}

Simonsen, Søren Bredmose; Chorkendorff, Ib; Dahl, Søren; Skoglundh, Magnus; Meinander, Kristoffer; Jensen, Thomas N.; Lauritsen, Jeppe Vang; Helveg, Stig

\section{Published in:}

The Journal of Physical Chemistry Part C

Link to article, DOI:

$10.1021 /$ jp2098262

Publication date:

2012

Document Version

Peer reviewed version

Link back to DTU Orbit

Citation (APA):

Simonsen, S. B., Chorkendorff, I., Dahl, S., Skoglundh, M., Meinander, K., Jensen, T. N., Lauritsen, J. V., \& Helveg, S. (2012). Effect of Particle Morphology on the Ripening of Supported Pt Nanoparticles. The Journal of Physical Chemistry Part C, 116(9), 5646-5653. https://doi.org/10.1021/jp2098262

\section{General rights}

Copyright and moral rights for the publications made accessible in the public portal are retained by the authors and/or other copyright owners and it is a condition of accessing publications that users recognise and abide by the legal requirements associated with these rights.

- Users may download and print one copy of any publication from the public portal for the purpose of private study or research.

- You may not further distribute the material or use it for any profit-making activity or commercial gain

- You may freely distribute the URL identifying the publication in the public portal 


\section{Effect of Particle Morphology on the Ripening of}

\section{Supported Pt Nanoparticles}

Søren B. Simonsen ${ }^{1,2}$, Ib Chorkendorff ${ }^{2}$, Søren Dahl ${ }^{2}$, Magnus Skoglundh ${ }^{3}$, Kristoffer Meinander ${ }^{4}$, Thomas N. Jensen ${ }^{4}$, Jeppe V. Lauritsen ${ }^{4}$, and Stig Helveg ${ }^{1, *}$

${ }^{1}$ Haldor Topsøe A/S, Nymøllevej 55, DK-2800 Kgs. Lyngby, Denmark, ${ }^{2}$ CINF, Department of Physics, Technical University of Denmark, DK-2800 Kgs. Lyngby, Denmark, ${ }^{3}$ Competence Centre for Catalysis (KCK), Chalmers University of Technology, SE-41296, Göteborg, Sweden and ${ }^{4}$ Interdisciplinary Nanoscience Center (iNANO), Aarhus University, DK-8000 Aarhus C, Denmark

*Address correspondence to phone: +45 4527 2000, fax: +45 4527 2999, e-mail: sth@topsoe.dk RECEIVED DATE

ABSTRACT: To improve the understanding of sintering in diesel and lean-burn engine exhaust aftertreatment catalysts, we examined oxygen-induced sintering in a model catalyst consisting of $\mathrm{Pt}$ nanoparticles supported on a planar, amorphous $\mathrm{Al}_{2} \mathrm{O}_{3}$ substrate. After ageing at increasing temperatures, transmission electron microscopy analysis reveals that highly monodisperse ensembles of nanoparticles transformed into ensembles with bimodal and subsequently Lifshitz-Slyozov-Wagner particle size distributions. Moreover, scanning transmission electron microscopy and atomic force microscopy analysis suggest that the Pt nanoparticles have size-dependent morphologies after sintering in oxidizing environment. The evolution of the particle sizes is described by a simple kinetic model for ripening and the size-dependent particle morphology is proposed as an explanation for the observed 
bimodal particle size distribution shapes.

KEYWORDS: sintering, Ostwald ripening, $\mathrm{Pt} / \mathrm{Al}_{2} \mathrm{O}_{3}$, TEM, STEM, AFM.

\section{INTRODUCTION}

The physicochemical properties of nanoparticles may depend on their size, morphology and composition ${ }^{1}$. For applications as a heterogeneous catalyst, the nanoparticle edges and kinks often play an important role for the catalytic activity and these sites are predominant for the smallest nanoparticles 1-3. To preserve the active sites and to avoid loss of the total surface area of the catalytically active phase, it is important to stabilize the supported nanoparticles, with sizes of a few nanometers, during operational conditions at often high temperatures in the relevant gas environments ${ }^{4}$. Although a variety of techniques allow well-defined nanoparticles to be synthesized, the nanoparticles are inherently unstable due to their high surface area and thus there is a thermodynamic drive for coarsening of the nanoparticles ${ }^{5-8}$.

Sintering of nanoparticles is indeed one main cause of catalyst deactivation and research aimed at understanding the fundamental nanoparticle sintering processes is needed to aid the development of catalysts with enhanced long-term stability. A vast amount of the published coarsening studies has

focused on the evolution of the nanoparticle sizes as a function of time and process conditions ${ }^{5-20}$. A prototypical nanocatalyst system for studying coarsening consists of Pt nanoparticles supported on an $\mathrm{Al}_{2} \mathrm{O}_{3}$ material, which is relevant as an oxidation catalyst in diesel and lean-burn engine exhaust aftertreatment technologies. Previous microscopy studies have demonstrated that Pt nanoparticles on $\mathrm{Al}_{2} \mathrm{O}_{3}$ coarsen via the Ostwald ripening mechanism in an oxidizing environment ${ }^{13,21,22}$. The enhanced sintering of Pt by oxygen has been attributed to the formation of volatile Pt-oxide species ${ }^{6-8,14}$. In the Ostwald ripening process, the atomic species are exchanged among immobile nanoparticles driven by a difference in the chemical potential of atoms in the nanoparticles, as described by the Gibbs-Thomson 
equation. Nanoparticles with a larger or smaller radius of curvature have a lower or higher chemical potential, respectively, which results in a net atom-transport from the smaller to the larger nanoparticles 5-8. In addition to the metal nanoparticle size, the morphology and metal-support interactions will also affect the radius of curvature, the surface-to-volume ratio and the interfacial perimeter between the particle and support. The morphology and metal-support interactions have therefore also been considered of potential importance for the sintering in heterogeneous catalysts 12,13,23-27. However, although Wynblatt and Gjostein in their pioneering work ${ }^{6}$ emphasized the impact of the morphology of the supported nanoparticles on their tendency for ripening, experimental studies of the relationship between the three-dimensional (3D) morphology and ripening of supported nanoparticles have been lacking.

In this paper we address how ripening of Pt nanoparticles supported on a planar, amorphous $\mathrm{Al}_{2} \mathrm{O}_{3}$ substrate is influenced by their three-dimensional (3D) morphology. The evolution in the shape of the particle size distribution (PSD) is determined by means of transmission electron microscopy (TEM) as a function of ageing temperature after thermal ageing in air. The 3D morphology of the nanoparticles was studied by scanning transmission microscopy (STEM) and atomic force microscopy (AFM) and is expressed in terms of the height-to-diameter ratio as a function of the nanoparticle size. The relationship between the sintering process, described statistically by the evolution in the PSD shape, and the sizedependent morphology of the nanoparticles is discussed in the light of a simple kinetic model for ripening. The observed evolution of the PSD shape is compared to simulations of PSDs by taking the size-dependent height-to-diameter ratio into account. The analysis is related to the previously reported time-dependent evolution in the PSD shape of $\mathrm{Al}_{2} \mathrm{O}_{3}$-supported Pt nanoparticles upon ageing ${ }^{21}$.

\section{EXPERIMENTAL METHODS}

The present model catalysts consisted of Pt nanoparticles dispersed on a flat, amorphous $\mathrm{Al}_{2} \mathrm{O}_{3}$ support. The catalysts were supported on $\mathrm{Si}$ wafers with amorphous and electron-transparent $\mathrm{Si}_{3} \mathrm{~N}_{4}$ 
windows. Further preparation details are described in ref. ${ }^{21}$.

A series of ageing experiments were carried out by exposing the $\mathrm{Pt} / \mathrm{Al}_{2} \mathrm{O}_{3}$ samples in tube furnace (Carbolite CFT) to $0.2 \% \mathrm{O}_{2}$ in $\mathrm{N}_{2}$ at a total pressure of 1 bar and a flow of $120 \mathrm{ml} / \mathrm{min}$ and to temperatures of 450, 550, 600 and $650{ }^{\circ} \mathrm{C}$. Specifically, for each experiment, a $\mathrm{Pt} / \mathrm{Al}_{2} \mathrm{O}_{3}$ sample was placed in a porcelain boat in the centre of the tube furnace. By using an ADM2000 universal gas flowmeter (Agilent Technologies), the gas flow was set at room temperature. After the establishment of the gas flow, the furnace was heated by $5{ }^{\circ} \mathrm{C} / \mathrm{min}$ until the ageing temperature was reached. The samples were kept 3 hours at the ageing temperature, whereupon the furnace was cooled, by turning off the furnace, at a rate of ca. $2{ }^{\circ} \mathrm{C} / \mathrm{min}$ for the first $200{ }^{\circ} \mathrm{C}$ and subsequently at a slower pace down to room temperature. The furnace temperature was measured with an estimated error of $\pm 7^{\circ} \mathrm{C}$ determined by inserting an additional thermocouple in the tube furnace near the sample in one experiment. After the cooling of the sample to room temperature, the gas flow measurement was confirmed.

The TEM examination was performed by using an image aberration corrected Titan 80-300 SuperTwin Environmental Transmission Electron Microscope (FEI Company) operated at a primary electron energy of $300 \mathrm{keV}$ with an information limit of ca. $0.10 \mathrm{~nm}$. TEM images were acquired using a bottom-mounted $2 \mathrm{k}$ x $2 \mathrm{k}$ charged-coupled device (CCD) camera at a magnification corresponding to pixel size of $0.09 \mathrm{~nm}$. From the TEM images, Pt particle sizes (diameters) were measured by using a circular approximation to their projected area. The measurements were performed automatically as described in ref. ${ }^{21}$. The estimated measuring error is a systematic error of $10 \%$ of the particle diameters due to calibration of the TEM and a random error of $0.5 \mathrm{~nm}$ for all particle diameters due to the automatic image analysis. For particle sizes below ca. $2 \mathrm{~nm}$ in diameter, the error due to the automatic measurements increases and becomes systematic. For this reason the presented data only include particle diameters above $2 \mathrm{~nm}$.

The STEM characterization was performed using the same Titan 80-300 electron microscope. The microscope was operated at a primary energy of $300 \mathrm{keV}$ and with an electron probe size less than 0.23 
nm, as calibrated towards the observation of the Pt(111) lattice fringes. A high-angle annular dark field (HAADF) detector was used to collect scattered electrons and ensure high contrast of the Pt nanoparticles. Images were recorded at a magnification corresponding to a pixel size of $0.16 \mathrm{~nm}$. Furthermore, all images were recorded with the same contrast and brightness level for proper comparison of different images. From the STEM images, the particle diameters were measured as described for the TEM images. The signal intensity of the nanoparticles was integrated over the projected area corresponding to each particle and the background signal contribution generated by the support material was subtracted.

Non-contact atomic force microscopy (NC-AFM) was performed at room temperature in an ultra-high vacuum (UHV) chamber with a base pressure below $1 \times 10^{-10}$ mbar using a beam deflection NC-AFM described in ${ }^{28}$. The very sensitive non-contact operation mode of the AFM was chosen in order to minimize tip-induced movements of the nanoparticles. The sample was fixed on a standard Ta sample plate and introduced to the UHV chamber through a vacuum load lock. No further sample processing was performed. The NC-AFM images were recorded in the topography mode by keeping the mean frequency shift $(\Delta f)$, due to the tip-surface interaction, constant relative to a preset frequency shift value and by recording the feedback signal of the tip-surface distance control as the tip traced the surface. A voltage was applied to the tip, relative to the sample holder, $U_{\text {bias, }}$ which was adjusted to minimize the electrostatic forces arising from the contact potential difference ${ }^{29}$. To optimize the AFM resolution and to reduce the risk of mechanically pushing the clusters, the high-aspect-ratio carbon nanotube (CNT) terminated silicon probes (Nanosensors, CNT-NCH type) with cantilever spring constants of ca. 42 N/m and resonance frequencies of ca. $330 \mathrm{kHz}$ were used ${ }^{30}$. Kelvin probe (KPFM) measurements, in which active regulation of the tip-sample bias voltage was included ${ }^{31}$, showed negligible effects on the height in topographical images.

Computer simulations of the temporal evolution of the PSDs were performed following the procedure of Smet et. al. ${ }^{32}$ based on the mean-field kinetic model for interface-controlled ripening ${ }^{6}$ : 
eq. 1

$$
\frac{d R}{d t}=\alpha \frac{\alpha^{\prime}}{R^{2}}\left(\frac{R}{R^{*}}-1\right)
$$

Here $\alpha$ is a geometrical factor that describes the morphology of each particle. Approximating the supported metal particle morphology by a spherical cap with a radius of curvature, $R$, and a metal-oxide contact angle, $\theta$, results in a geometrical factor $\alpha=\sin (\theta) /\left(1 / 2-3 / 4 \cos (\theta)+1 / 4 \cos ^{3}(\theta)\right)$. $\alpha^{\prime}$ describes the system specific parameters, such as the diffusion energy barrier for atomic species to cross the metalsupport interface as well as the metal surface energy, the atomic volume and the temperature. $R^{*}$ is the critical radius of curvature corresponding to the particle size which is in equilibrium with the mean-field concentration of diffusing atomic species ${ }^{6,20}$. Since $R^{*}$ equals the mean radius of curvature ${ }^{33}$, this parameter is equaled to the arithmetic mean of $R$ for the particle ensemble in the simulations. Simulations were also performed based on the mean-field kinetic model including a particle morphology consisting of a spherical cap resting on a cylindrical metal layer at the interface between the spherical cap and the oxide support. With this morphology model, eq. 1 becomes,

eq. 2

$$
\frac{d R}{d t}=\frac{\sin (\theta) \alpha^{\prime}}{2 R^{2}\left(1 / 2-3 / 4 \cos (\theta)+1 / 4 \cos ^{3}(\theta)\right)+H R \sin ^{2}(\theta)}\left(\frac{R}{R^{*}}-1\right)
$$

where $R$ is the radius of curvature, $\theta$ the contact angle between the spherical cap and the interface layer, $H$ the height of the interface layer, $R^{*}$ the critical radius of curvature and $\alpha^{\prime}$ describes the system specific parameters. In the simulations based on eq. 2, the effect of the interface layer was described by $\theta=0.6 \pi$ and $H=0.7 \mathrm{~nm}$. For comparison with the TEM data, the radii of curvature, $R$, were converted to the projected diameters as $d=2 R$, because the results show that $\theta>\pi / 2$ for all particles. The PSD of the as-prepared $\mathrm{Pt} / \mathrm{Al}_{2} \mathrm{O}_{3}$ sample (Fig. 1f) was used as the initial PSD. For all simulations, the 
calculation steps corresponded to the time units, $\alpha^{,-1} \cdot \mathrm{nm}^{3}$. Simulations were performed in an iterative way by varying $\alpha$, until the simulated mean diameter for the last simulation step equaled the observed mean diameter after ageing at $650{ }^{\circ} \mathrm{C}$.

\section{RESULTS AND DISCUSSION}

Figure 1a-e shows TEM images of the as-prepared and the aged samples. In the TEM images, the Pt nanoparticles are identified as the darker contrast features and the planar, amorphous $\mathrm{Al}_{2} \mathrm{O}_{3}$ support is associated with the brighter areas. In the as-prepared state, the Pt nanoparticles appear almost monodispersed with a homogeneous distribution over the support area (Fig. 1a). A visual inspection of the TEM images reveals an increase of the mean particle size and a reduction of the particle density for temperatures above $600{ }^{\circ} \mathrm{C}$ (Fig. 1b-e), in agreement with previous studies ${ }^{5,7,8,11,14,18,22,34,35}$. Furthermore, Figure 1f-j presents PSDs corresponding to the ageing conditions in Figure 1a-e. In the as-prepared samples, the Pt nanoparticles have a mean projected diameter of $2.7 \mathrm{~nm}$. The ageing treatments did not significantly alter the PSD shape up to $600{ }^{\circ} \mathrm{C}$. However, after ageing at $600{ }^{\circ} \mathrm{C}$, the PSD is asymmetric with a noticeable shoulder to the large particle side of the main peak (Fig. 1i). After ageing at $650{ }^{\circ} \mathrm{C}$, the asymmetric PSD shape has flipped over so that the main peak is associated with a tail towards the smaller particle sizes (Fig. 1j).

Ripening is often described by the so-called Lifshitz-Slyozov-Wagner (LSW) model ${ }^{36,37}$. A LSW model, relevant for a two-dimensional system ${ }^{38}$, fits the PSD shape at $650{ }^{\circ} \mathrm{C}$ in Fig. $1 \mathrm{j}$ well. The agreement is indeed expected since the $\mathrm{Al}_{2} \mathrm{O}_{3}$ support is planar and homogeneous and so closely matches the assumptions of the LSW model. Thus, the PSD shape that emerges after ageing at $650{ }^{\circ} \mathrm{C}$ is therefore consistent with the ripening mechanism. Our previous time-resolved in situ TEM study of the present type of $\mathrm{Pt} / \mathrm{Al}_{2} \mathrm{O}_{3}$ model catalyst in 10 mbar air and at $650{ }^{\circ} \mathrm{C}$ showed directly that Pt sintering is governed by ripening and that ripening leads to a temporal evolution of the PSD shape similar to the temperature-dependent progress presented in Figure $1 \mathrm{f}-\mathrm{j}^{21}$. Specifically, as a function of ageing time, 
the initial monodispersed PSD shape transforms into a transitional shape, characterized by the appearance of a shoulder to the large particle side of the main peak, and subsequently into a shape, characterized by LSW model with a tail to the small particle side of the main peak ${ }^{21}$. The present ex situ and the previous in situ results therefore indicate that ripening can cause a transformation of an initial narrow PSD into the LSW-shape. However, the shape of LSW-distribution will obviously not fit the PSD shape at $600{ }^{\circ} \mathrm{C}$ (Fig. 1i) and the observed bimodal-like shape is in fact unexpected for kinetic ripening models such as eq. $1^{32,39}$. Equation 1 is based on additional assumptions such as a mean-field concentration of diffusing species and a size-invariant particle morphology. Because the mean-field assumption can not account for the bimodality ${ }^{32,39}$, the relationship between the nanoparticle morphology and PSD evolution will be addressed in the following.

To estimate the morphology of the individual Pt nanoparticles, STEM images of the samples were acquired. Figure 2a presents a STEM image of the sample after thermal ageing at $650{ }^{\circ} \mathrm{C}$. The particles can be identified as the bright area projections and the oxide support corresponds to the larger black area. The STEM images provide information about the nanoparticle's diameter as well as their volume. The latter is proportional to the image intensity associated with a nanoparticle ${ }^{40,41}$. Figure $2 \mathrm{~b}$ shows the intensity versus diameter for the individual nanoparticles in STEM images and allows any sizedependent variation in the morphology of the supported Pt nanoparticles to be addressed, because the exact 3D nanoparticle shape determine the volume-diameter relationship. Since the exact 3D shape of the $\mathrm{Al}_{2} \mathrm{O}_{3}$-supported Pt nanoparticles is not known a priori, a spherical cap is here used as a tentative approximation to the nanoparticle morphology. Moreover, the particle morphology is distinguished from the particle shape in the sense that roughly identical particle morphologies may be terminated by different crystallographic facets that are unique for different particle shapes. The spherical cap is uniquely defined by just one descriptor, namely by a metal-support contact angle, $\theta$, or equivalently, by the ratio of the particle height and diameter, $h / d$ (defined in the insert of Figure 2a). The volume of a spherical cap with a constant height-to-diameter ratio as a function of the particle size was fitted to the 
experimental observations in Figure 2b (dashed black) by varying the STEM intensity calibration constant C (see caption Fig. 2) and morphology factor until the total squared difference between the modeled and measured data is minimized, resulting in $R^{2}=0.95$. Figure $2 \mathrm{~b}$ shows that the volume of a spherical cap with a constant morphology increases faster with the diameter than the volume of the observed Pt nanoparticles. The morphology of the observed particles is therefore not fully consistent with a spherical cap with a constant height-to-diameter ratio for all particle sizes. One explanation for this difference is that the height-to-diameter ratio for the observed particles decreases with increasing particle diameter. However, because the STEM intensity calibration constant $C$ is unknown, the true relation between the particle height and diameter cannot be determined based on only Figure $2 \mathrm{~b}$.

To directly measure the absolute height of the nanoparticles, the model catalyst aged $650{ }^{\circ} \mathrm{C}$ was studied by AFM. Figure 3a shows such an image in which the AFM tip height relative to the oxide support is depicted by a grey scale in such a way that the Pt nanoparticles are identified as brighter protrusions on the darker background corresponding to the $\mathrm{Al}_{2} \mathrm{O}_{3}$ support. The significantly larger (> 20 $\mathrm{nm}$ ), bright features represent impurities on the surface, possibly induced during sample transfer to the UHV chamber of the AFM. Pt nanoparticles at or in close vicinity of such regions were avoided in the data analysis. By measuring the height and width of all particles in the AFM images, the cluster dimensions were obtained and presented in Fig. 3 as the particle height distribution (PHD) (b) and the $\operatorname{PSD}(\mathrm{c})$

The particle diameters obtained from AFM (Fig. 3c) are generally considerably larger relative to the diameters as obtained from TEM (Fig. 1j). This difference can be attributed to the well-known effect of tip broadening in AFM for objects with a size comparable to the tip apex dimensions. Therefore, in order to obtain a direct correlation plot of the nanoparticle heights vs. the diameters, the PSD obtained from AFM was aligned with the analogous PSD from TEM. The alignment was performed by minimization of the total squared difference between the column heights in the PSDs from AFM and TEM. The minimization was obtained, with the reduced $\chi^{2}=$ ca. 1 , by the multiplication by a factor 0.7 
and the subtraction of the value $2.4 \mathrm{~nm}$ (Figure 4a). The effect of AFM tip broadening is graphically illustrated in figure $4 \mathrm{~b}$ where circles are superimposed on the particle positions in a TEM image and have diameters corresponding to calculated, non-calibrated AFM diameters. Figure 4b serves to illustrate that the broadening is sufficiently small to allow the detection of small particles in the vicinity of larger ones in the AFM images. Figure 4c presents the final plot for the particle heights as a function of the nanoparticle diameters corrected for tip broadening. The relatively large spread in the particle heights for specific diameters is probably due to the relatively rough method of deconvolution of the AFM tip broadening by the statistical alignment of the AFM data to the TEM data. For this reason the AFM data should only be used to indicate the overall trend between the particle heights and diameters. Interestingly, the majority of the data points lies in the region between the lines corresponding to a spherical (solid grey) and a hemispherical (dashed grey) particle morphology (Fig. 4c). The $\mathrm{Al}_{2} \mathrm{O}_{3^{-}}$ supported Pt nanoparticles are therefore generally flatter than spheres, but higher than hemispheres. Assuming a linear height-to-diameter relation as a first approximation, two straight lines were fitted to the data: One which was unconstrained (solid black) for which $R^{2}=0.48$ and one which was constrained through the origin (dashed black) for which $R^{2}=0.46$. From the fitted lines shown in Figure $4 \mathrm{c}$, the sizedependent height-to-diameter ratio was calculated and presented in Figure 4d (solid black and dashed black). The unconstrained fitted line corresponds to a height-to-diameter ratio that indeed decreases as a function of particle size, reflecting a size-dependent Pt nanoparticle morphology. The AFM data does not describe the height-to-diameter ratio for particle sizes below 2 nm (Fig. 4c-d). Extending the unconstrained fit in Figure 4c below the limit of $2 \mathrm{~nm}$ will result in a height-to-diameter ratio $>1$. As this ratio seems to contradict usual observations of supported nanoparticles, it is more likely that the height-to-diameter ratio is constant for the smallest particle sizes, or perhaps, that the height-to-diameter ratio decreases due to effects of support defect sites. However, the 3D morphology of the smallest particles cannot be determined from the present data. For the constrained fit, the line corresponds to a constant height-to-diameter ratio for all particle sizes. Since both the unconstrained and the constrained 
line fits describe the data almost equally well, the AFM results indicate a size-dependence of the heightto-diameter ratio only as a possibility. For comparison with the STEM data, the volume of a spherical cap including the size-dependent height-to-diameter ratio, corresponding to the unconstrained fitted line in Figure 4d, was fitted to Figure 2b (solid black), resulting in $R^{2}=0.96$. Similarly, the constrained fitted line in Figure 4d corresponds to the previously fitted volume of a spherical cap with constant height-to-diameter ratio (Figure 2b (dashed black)). The nanoparticle volume, including the sizedependent height-to-diameter ratio, fits the STEM data slightly better than the fitted volume corresponding to the constant nanoparticle morphology. The difference between the two fits based on the AFM data seems relatively small (Fig. 2b), compared to the trend of the STEM data which indicates a stronger size-dependence of the nanoparticle morphology (Fig. 4c). It is emphasized that this discrepancy is not due to the error of the alignment of the AFM data to the TEM data, since no multiplication and subtraction values applied in the alignment procedure results in a close match of the AFM data both to the TEM and STEM data. To demonstrate the stronger size-dependence as observed by STEM, the volume of a spherical cap was again fitted to the STEM data (Fig. 2b), assuming a linear relation between the height and diameter, but in this case with an unconstrained slope and a $h / d=1$ for $d$ $=2 \mathrm{~nm}$, consistent with the unconstrained fit to the AFM data. The fit was optimized by varying the slope of the linear height-to-diameter relation until the total squared difference between the modeled and measured data points was minimized, resulting in the linear relation $h=0.3 d+1.4 \mathrm{~nm}$.. The resulting fit to the STEM data has $R^{2}=0.99$ and is indicated by the black dotted line in Figure 2b. The stronger sizedependence of the height-to-diameter relation according to the STEM data can be appreciated by including the black dotted line from Figure $2 \mathrm{~b}$ in Figure 4d. In summary, the combined STEM and AFM data suggests that the $\mathrm{Al}_{2} \mathrm{O}_{3}$-supported Pt nanoparticles generally are flatter than spheres and that the height-to-diameter ratio decreases with increasing particle size, although the two data sets differ on the degree of the size-dependence of the height-to-diameter ratio. The size-dependent height-to-diameter ratio reflects an increasing wetting of the support by Pt, corresponding to an increasing work of 
adhesion with increasing size of the Pt nanoparticles in the present oxidizing environment.

A size-dependent morphology of the supported Pt nanoparticles may influence the ripening process in several ways. Firstly, the observed growth or shrinkage of the projected nanoparticle areas in the TEM images depends on the nanoparticle volume which is described in terms of the height-to-diameter ratio. Secondly, different nanoparticle morphologies imply that the nanoparticle perimeter at the Pt-oxide interface, and thus the total number of sites that can emit or absorb diffusing species, varies. Thirdly, the nanoparticle morphology influences the concentration of atomic species, which depends on nanoparticle surface curvature (the Gibbs-Thomson relation). To relate the observed size-dependent particle morphology to the sintering process, the results in Figure 1 are compared to computer simulations based on Eq. 1. Two set of simulations were performed: In the first simulation, $\alpha$ was set to capture the main trends of the size-dependent height-to-diameter ratio, resulting from the unconstrained linear fit to the AFM data, by using a continuous height-to-diameter-trace corresponding to the grey dotted line in fig. 4d. This trace, which resembles the AFM data rather than the STEM data, was chosen to examine the effect of the least size-dependent variations in the height-to-diameter ratio. In the second simulation, $\alpha$ had a constant value identical to the dashed black line in Figure 4d, corresponding to the constrained linear fit to the AFM data. Figure 5a-c presents the simulated PSDs based on the size-dependent heightto-diameter ratio. Here, the initial PSD evolves via transitional bimodal shapes, which are characterized by an additional peak towards the larger particle sizes, into a shape of the LSW-type, which has a main peak shifted towards the large particle sizes and a tail towards the small sizes (Fig. 5a-c). As time and temperature are linked in Eq. 1, simulations of the change in the PSD shape with increasing time also match the simulated change with increasing temperatures. Thus it is intriguing that the simulated evolution in the PSD shape matches the experimental observations. Similarly, the simulated PSD shapes match the experimental time-dependent observations in our previous in situ TEM study of the same type of $\mathrm{Pt} / \mathrm{Al}_{2} \mathrm{O}_{3}$ model catalyst ${ }^{21}$. For comparison, Figure $5 \mathrm{~d}-\mathrm{f}$ presents the simulated PSDs based on the constant height-to-diameter ratio. In this case, the PSDs are characterized by a single peak that gradually 
shifts toward larger nanoparticle sizes and by the appearance of a tail toward smaller nanoparticle sizes, as the ageing time increases. The transition of the PSD toward the LSW-shape does not indicate any development of an additional peak. Thus, according to the simulations in Figure 5, the additional peak in the PSD therefore only develops for particles with a size-dependent height-to-diameter ratio. As this ratio decreases with increasing particle diameter, ripening results in an effectively larger fraction of particles with a larger projected diameter in the TEM images compared to an ensemble of particles with a size-independent height-to-diameter ratio, which may explain the emergence of the additional peak.

In the spherical cap approximation for the 3D nanoparticle morphology, a size-dependence of the height-to-diameter ratio corresponds to a size-dependent degree of wetting. It has previously been reported that defects in an alumina surface can influence the morphology of the supported nanoparticles 27. Although the present model catalysts are assumed to be planar and amorphous, surface defects cannot be excluded and a physical reason for a size-dependent wetting could therefore be speculated to result from such support defects. The present observations do, however, not directly address this possibility. Moreover, describing the nanoparticle morphology by a single parameter, such as the contact angle, alone may be an oversimplified approximation and morphologies described by additional parameters could be considered. One such example is the spherical cap combined with a metal layer in the interface between the spherical cap and the oxide support. The existence of such an interface layer has been reported for Cu-supported Ag nanoparticles ${ }^{42}$. With this model the observed size-dependence of the height-to-diameter ratio can be described by a constant height of the interface layer while maintaining a constant contact angle. The presented STEM (Fig. 2b) and AFM (Fig. 4c) data could be fitted equally well using this two-parameter morphology description (where the best fit to the AFM data resulted in $\theta=0.6 \pi$ and $H=0.7 \mathrm{~nm}$, Eq. 2) as using the single parameter description. Similarly, additional simulations based on the two-parameter model (Eq. 2, with $\theta=0.6 \pi$ and $H=0.7 \mathrm{~nm}$ ) reproduce the trends in the evolution of the PSD shape as found for the single parameter description, although the additional peak towards the large particle side of the main peak is less pronounced. The 
presence of such alternative nanoparticle morphologies in the present model catalysts cannot be excluded and it will require further nano-scale tomographic investigations in order to determine the 3D nanoparticle morphology in more detail. Moreover, the morphology estimation based on STEM may also be slightly affected by a size-dependent Pt surface oxidation of the nanoparticles ${ }^{43,44}$ that would result in a size-dependent change of the mass-thickness contrast. Although, the chemical state of the Pt nanoparticles was not determined, a Pt oxidation would likely have been confined to the surface region under the present conditions ${ }^{13}$ and thus the effect on the mass-thickness contrast should be limited.

\section{CONCLUSIONS}

By means of TEM, STEM and AFM measurements, we have shown that alumina-supported Pt nanoparticles sintered and adopted size-dependent particle morphologies in an oxidizing environment. The relationship between progress in the particle size distribution (PSD) and the size-dependent particle morphology is discussed in the light of a kinetic model for ripening. It is shown that the evolution of monodisperse ensembles of Pt nanoparticles into ensembles described by a LSW-shaped PSD is characteristic for the ripening model and that the appearance of transitional bimodal PSDs can be explained by the size-dependent 3D morphology of the supported Pt nanoparticles. The results suggest that statistical descriptions of sintering phenomena, in terms of PSDs, are sensitive to a size-dependence of the nanoparticle morphology and so, in general, the stability of supported nanoparticles may thus depend on both their size and shape.

\section{ACKNOWLEDGEMENT}

We gratefully acknowledge Bengt Kasemo, Jonas Andersson, Elin Larsson and Laurent Feuz (Chemical Physics Group) as well as Eva Olsson (Microscopy and Microanalysis Group) at Chalmers University of Technology for contributing to sample preparations. We thank the MC2-Access project for financial support. We acknowledge the participation of the CTCI Foundation, Taiwan, in the establishment of the 
in situ TEM facility at Haldor Topsøe A/S. CINF is funded by The Danish National Research

Foundation. JVL, KM and TNJ gratefully acknowledge financial support from the European Research Council (ERC starting grant no. 239834).

\section{REFERENCES}

1 Li, Y.; Somorjai, G. A. Nanolett. 2010, 10, 2289-2295.

2 Nørskov, J. K.; Bligaard, T.; Abild-Pedersen, F.; Chorkendorff, I.; Christensen, C. H. Chem. Soc. Rev. 2008, 37, 2163-2171.

3 Jones, G.; Jakobsen, J. G.; Shim, S. S.; Kleis, J.; Andersson, M. P.; Rossmeisl, J.; Abild-Pedersen, F.; Bligaard, T.; Helveg, S.; Hinnemann, B.; Rostrup-Nielsen, J. R.; Chorkendorff, I.; Sehested, J.; Nørskov, J. K. J. Catal. 2008, 259, 147-160.

4 Moulijn, J. A.; Diepen, A. E. v.; Kapteijn, F. Activity Loss. In Handbook of Heterogeneous Catalysis; Ertl, G.; Knözinger, H.; Schüth, F.; Weitkamp, J.; Eds., $2^{\text {nd }}$ Edition., Vol. 4, Wiley-VCH Verlag GmbH \& Co KGaA, Weinheim, 2008; pp 1829-1871.

5 Flynn, S. E.; Wanke, P. C. Catal. Rev. Sci. Eng. 1975, 12, 93-135.

6 Wynblatt, P.; Gjostein, N. A. Prog. Solid State Ch. 1976, 9, 21-58.

7 Baker, R. T. K.; Bartholomew, C. H.; Dadyburjor, D. B. Sintering and Redispersion: Mechanisms and kinetics. In Stability of Supported Catalysts: Sintering and Redispersion; Horsley, J. A.; Ed.; Catalytica Inc: Mountain view, California, 1991; pp 169-225.

8 Bartholomew, C. H. Catalysis - A Specialist Periodical Report, volume 10; The Royal Society of Chemistry: Cambridge, 1993; pp 41-82.

9 Nakamura, M.; Yamada, M.; Amano, A. J. Catal. 1975, 39, 125-133. 
10 Granqvist, C. G.; Buhrman, R. A.; J. Catal. 1976, 42, 477-497.

11 Lee, T. J.; Kim, Y. G. J. Catal. 1984, 90, 279-291.

12 Harris, P. J. F. J. Catal. 1986, 97, 527-542.

13 Rickard, J. M.; Genovese, L.; Moata, A.; Nitsche, S. J. Catal. 1990, 121, 141-152.

14 Lööf, P.; Stenbom, B.; Nordén, H.; Kasemo, B. J. Catal. 1993, 144, 60-76.

15 Fuentes, G. A.; Salinas-Rodríguez, E. Realistic Particle Size Distributions during Sintering by Ostwald Ripening. In Studies in Surface Science and Catalysis, volume 139; Spivey, J. J.; Roberts, G. W.; Davis, B. H.; Eds.; Elsevier Science B. V., Amsterdam, 2001; pp 503-510.

16 Sehested, J.; Carlsson, A.; Janssens, T. V. W.; Hansen, P. L.; Datye, A. K. J. Catal. 2001, 197, 200-209.

17 Campbell, C. T.; Parker, S. C.; Starr, D. E. Science 2002, 298, 811-814.

18 Datye, A. K.; Xu, Q.; Kharas, K. C.; McCarty, J. M. Catal. Today 2006, 111, 59-67.

19 Yang, F; Chen, M. S.; Goodman, D. W. J. Phys. Chem. C 2009, 113, 254-260.

20 Houk, L. R.; Challa, S. R.; Grayson, B.; Fanson, P.; Datye, A. K. Langmuir 2009, 25, $11225-$ 11227.

21 Simonsen, S. B.; Chorkendorff, I.; Dahl, S.; Skoglundh, M.; Sehested, J.; Helveg, S. J. Am. Chem. Soc. 2010, 132, 7968-7975.

22 Baker, R. T. K.; Thomas, C.; Thomas, R. B. J. Catal. 1975, 38, 510-513.

23 Arai, M.; Ishikawa, T.; Nakayama, T.; Nishiyama, Y. J. Colloid Interf. Sci. 1984, 97, 254-265.

24 Wang, T.; Lee, C.; Schmidt, L. D. Surf. Sci. 1985, 163, 181-197. 
25 Lee, W. H.; Vanloon, K. R.; Petrova, V.; Woodhouse, J. B.; Loxton, C. M.; Masel, R. I. J. Catal. 1990, 126, 658-671.

26 Hansen, P. L.; Wagner, J. B.; Helveg, S.; Rostrup-Nielsen, J. R.; Clausen, B. S.; Topsøe, H. Science 2002, 295, 2053-2055.

27 Kwak, J. H.; Hu, J.; Mei, D.; Yi, C.-W.; Kim, D. H.; Peden, C. H. F.; Allard, L. F.; Szanyi, J. Science 2009, 325, 1670-1673.

28 Enevoldsen, G. H.; Foster, A. S.; Christensen, M. C.; Lauritsen, J. V.; Besenbacher, F. Phys. Rev. B 2007, 76, 205415.

29 Gritschneder, S.; Namai, Y.; Iwasawa, Y.; Reichling, M. Nanotechnology 2005, 16, S41-S48.

30 Barwich, V.; Bammerlin, M.; Baratoff, A.; Bennewitz, R.; Guggisberg, M.; Loppacher, C.; Pfeiffer, O.; Meyer, E.; Güntherodt, H.-J.; Salvetat, J.-P.; Bonard, J.-M.; Forró, L. Appl. Surf. Sci. 2000, 157, 269-273.

31 Sadewasser, S.; Lux-Steinger, M. C. Phys. Rev. Lett. 2003, 91, 266101.

32 Smet, Y. D.; Deriemaeker, L.; Finsy, R. Langmuir 1997, 13, 6884-6888.

33 Finsy, R. Langmuir 2004, 20, 2975-2976.

34 Ruckenstein, E.; Chu, Y. F. J. Catal. 1979, 59, 109-122.

35 Harris, P. J. F. Int. Mater. Rev. 1995, 40, 97-115.

36 Lifshitz, I. M.; Slyozov, V. V. J. Phys. Chem. Solids 1961, 19, 35-50.

37 Wagner, C. Z. Elektrochemie 1961, 65, 581-591.

38 Rogers, T. M.; Desai, R. C. Phys. Rev. B 1989, 39, 11956-11964. 
39 Coughlan, S. D.; Fortes, M. A. Scripta. Metall. Mater., 1993, 28, 1471-1476.

40 Carlsson, A.; Puig-Molina, A.; Janssens, T. V. W. J. Phys. Chem. B 2006, 110, 5286-5293.

41 Li, Z. Y.; Young, N. P.; Vece, M. D.; Palomba, S.; Palmer, R. E.; Bleloch, A. L.; Curley, B. C.; Johnston, R. L.; Jiang, J.; Yuan, J. Nature 2008, 451, 46-48.

42 Yeadon, M.; Yang, J. C.; Ghaly, M.; Averback, R. S.; Gibson, J. M. Mater. Sci. Eng. B 1999, 67, 76-79.

43 Wang, C.-B.; Lin, H.-K.; Hsu, S.-N.; Huang, T.-H.; Chiu, H.-C. J. Mol. Catal. A 2002, 188, 201208.

44 McCabe, R. W.; Wong, C.; Woo, H. S. J. Catal. 1988, 114, 354-367. 

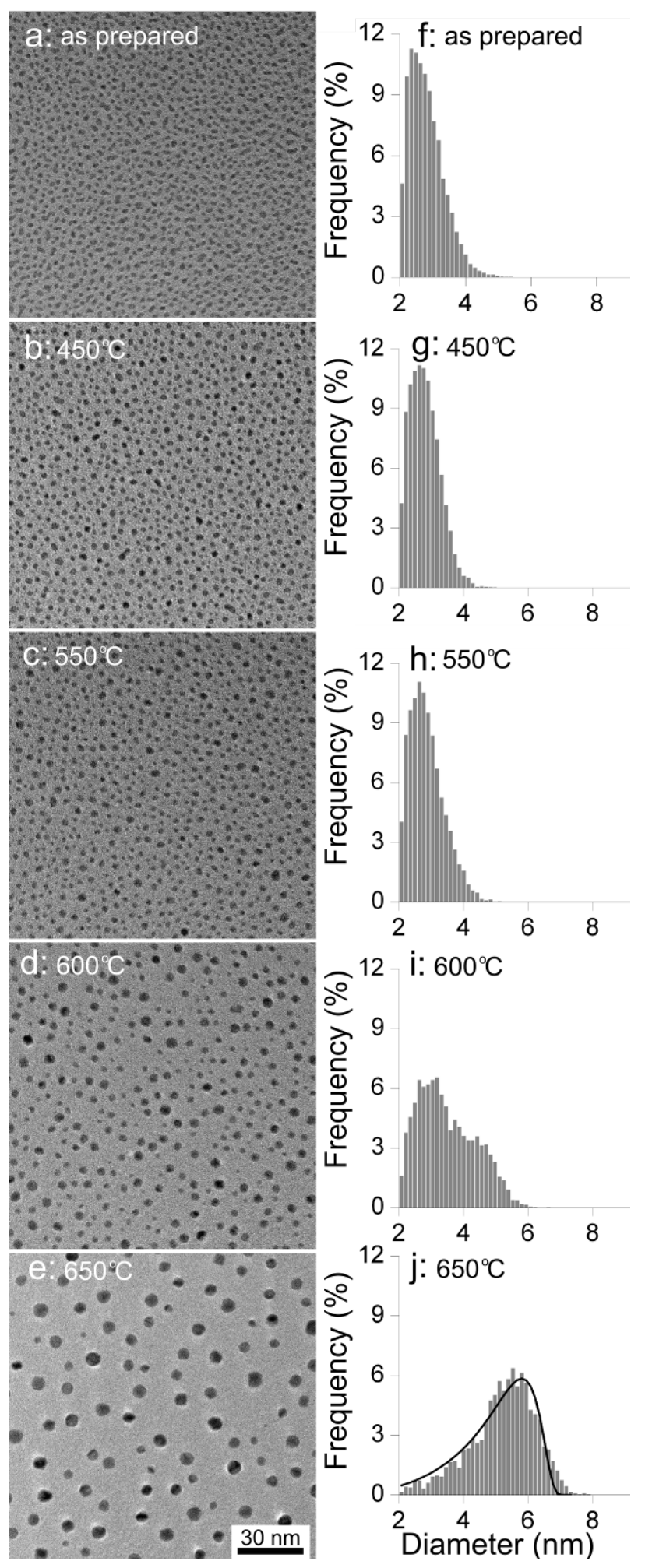

Fig. 1. (a-e) Representative TEM images of the $\mathrm{Pt} / \mathrm{Al}_{2} \mathrm{O}_{3}$ samples after ageing in a tube furnace at $1 \mathrm{bar}$ $0.2 \% \mathrm{O}_{2}$ in $\mathrm{N}_{2}$ for 3 hours and at 450, 550, 600 and $650^{\circ} \mathrm{C}$, respectively. (f-j) Particle size distributions based on measurements from a larger number of TEM images. The number of measured particles are (f) 14243, (g) 11204 (h) 8310, (i) 7606 and (j) 2954. In (j), the black curve shows a fit of a two-dimensional LSW distribution function ${ }^{32}$ to the particle size distribution. 



Fig. 2. (a) A representative STEM image of the $\mathrm{Pt} / \mathrm{Al}_{2} \mathrm{O}_{3}$ sample after ageing in a tube furnace in 1 bar $0.2 \% \mathrm{O}_{2}$ in $\mathrm{N}_{2}$ for 3 hours at $650{ }^{\circ} \mathrm{C}$. The insert illustrates a supported Pt nanoparticle in profile and defines the geometrical parameters: The curvature of radius, $R$; the particle diameter, $d$; the particle height, $h$; and the metal-support contact angle, $\theta$. (b) The STEM signal intensity integrated over the projected area of the particles plotted in arbitrary units against the particle diameter, $d$ (grey dots). A fit of spherical cap volume, $V(d)=C \cdot\left[1 / 2-3 / 4 \cos (\theta)+1 / 4 \cos ^{3}(\theta)\right] \cdot 1 / 6 \pi d^{3}$, to the data with $R^{2}=0.95$ is shown (dashed line). $C$ is a calibration factor for converting particle volumes to the STEM signal intensities. $\theta$ is kept constant for all particle sizes (dashed line). In addition, a plot is shown of $V(d)$ with a size-dependent $\theta$, corresponding to the size-dependent height-to-diameter ratio presented in Figure $4 d$, resulting in $R^{2}=0.96$ (solid line). Finally, a fit of $V(d)$ to the data is shown with a size-dependent $\theta$, assuming a linear relation between the particle height and diameter and a spherical morphology for particles with $d=2$, resulting in $R^{2}=0.99$ (dotted line). All fits are based on data points corresponding to diameters above $2 \mathrm{~nm}$. 

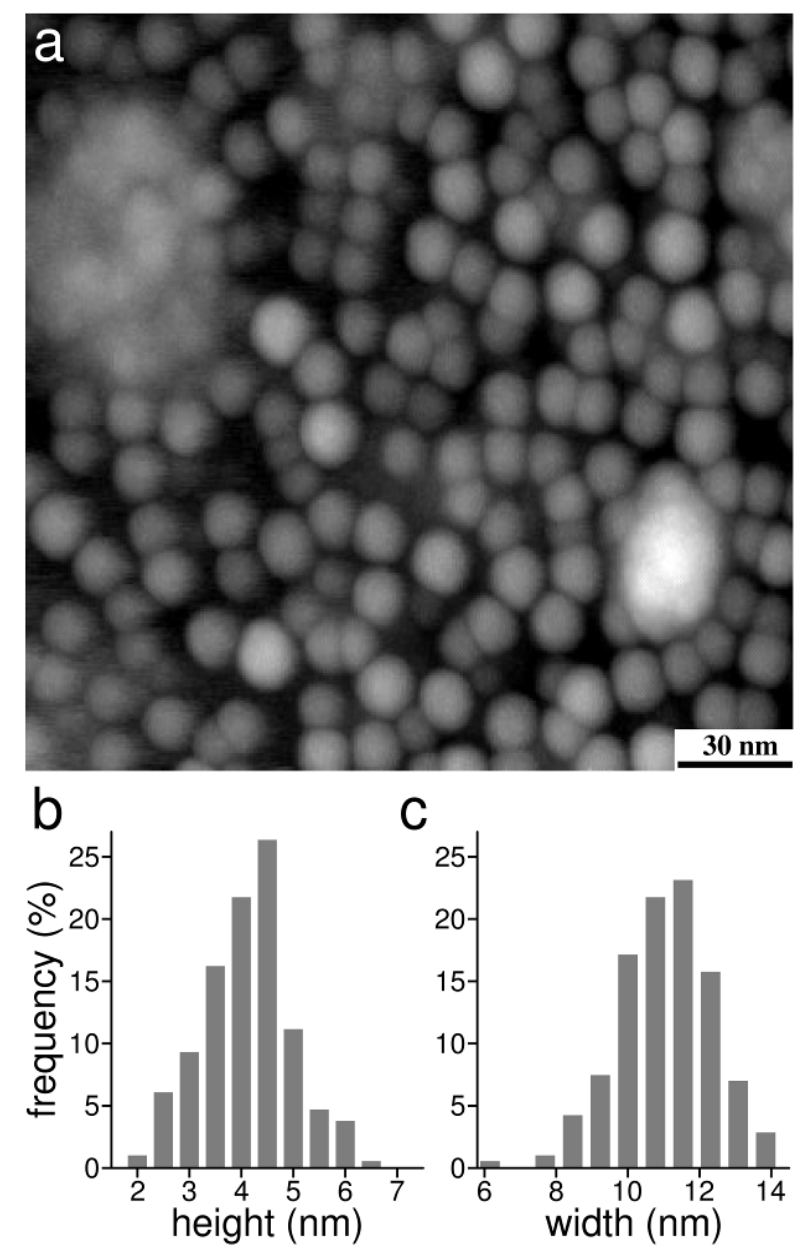

Fig. 3 (a) A representative AFM image of the $\mathrm{Pt} / \mathrm{Al}_{2} \mathrm{O}_{3}$ sample after ageing in a tube furnace in 1 bar 0.2 $\% \mathrm{O}_{2}$ in $\mathrm{N}_{2}$ for 3 hours at $650{ }^{\circ} \mathrm{C}$. (b) Particle height distribution and (c) particle size distribution based on measurements from a number of AFM images. The number of measured particles is 217. 

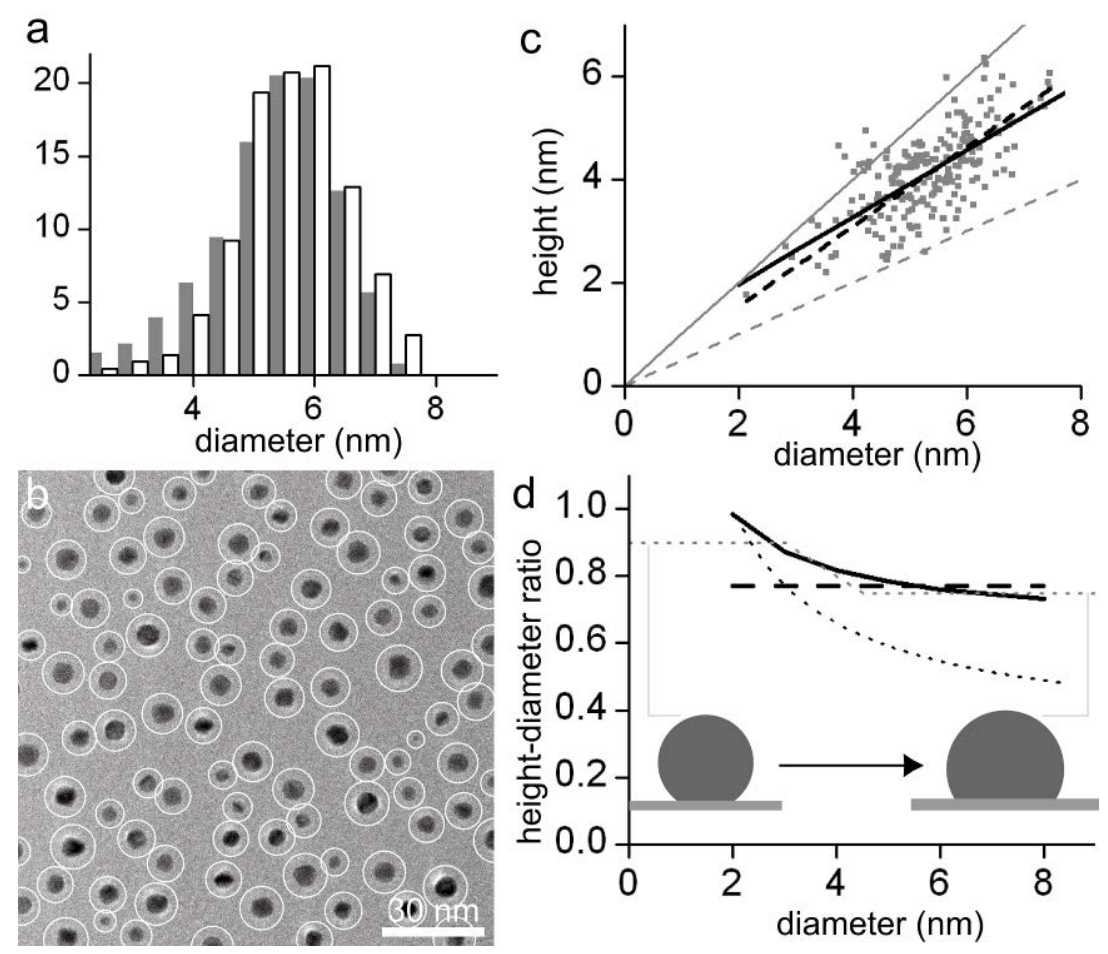

Fig. 4. (a) Particle size distributions from the calibrated AFM measurements (white) and TEM measurements (grey). (b) TEM image of the $\mathrm{Pt} / \mathrm{Al}_{2} \mathrm{O}_{3}$ sample after ageing at $650{ }^{\circ} \mathrm{C}$. The circles indicate the none-calibrated AFM diameters. (c) The nanoparticle height presented as a function of the calibrated nanoparticle diameter. Two straight lines represent fits to the dataset: One without any constraints (solid black) and one constrained to include the origin (dashed black). For comparison, lines corresponding to a spherical (solid grey) and a hemispherical (dashed grey) particle morphology are indicated. (d) The height-to-diameter ratio is presented as a function of nanoparticle size based on the unconstrained (solid black) and the constrained (dashed black) fitted lines in (c). The black dotted line corresponds to the black dotted line in Figure $2 \mathrm{~b}$ and represents the best fit to the STEM data. The height-to-diameter ratio values used in the simulation are indicated (dotted grey) and the nanoparticle morphologies corresponding to the highest and lowest values of the height-to-diameter ratio are illustrated. 

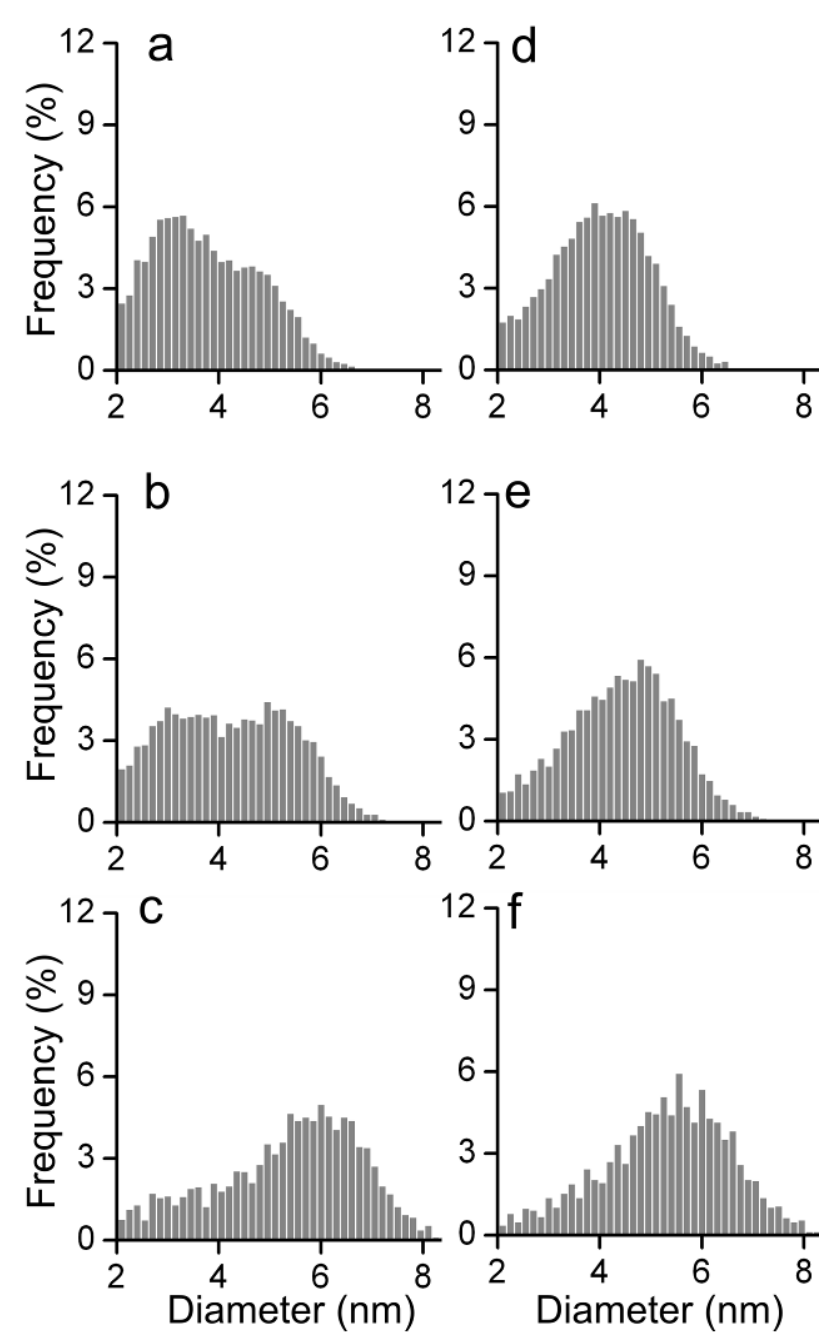

Fig. 5. Simulated particles size distributions based on Eq. 1 for particles with a decreasing (a-c) and a constant (d-f) height-to-diameter ratio. In the simulations, the metal-support contact angle $\theta$ (Eq. 1) is obtained from the height-to-diameter ratio indicated by (a-c) the grey dotted or (d-f) the black dashed line in Figure 4d. The number of calculation steps for each simulation, corresponding to the sintering time in arbitrary time units, is (a, d) 20, (b, e) 30 and (c, f) 60. The initial distributions are identical to the initial distribution of the $\mathrm{Pt} / \mathrm{Al}_{2} \mathrm{O}_{3}$ sample (fig. 1f). The number of particles in each distribution is (a) 6651, (b) 5220, (c) 2807, (d) 5439, (e) 4208 and (f) 2570. 